\title{
Banco de sementes do solo de sítios de Caatinga sob influência do Projeto de Integração do Rio São Francisco
}

Seed bank of Caatinga sites of under the influence of Integration Project San Francisco River.

\author{
J. R. Fabricante ${ }^{1 *}$; K. C. T. Araújo ${ }^{2}$; R. A. Castro ${ }^{2}$; V. M. Cotarelli ${ }^{2}$ \\ ${ }^{1}$ Departamento de Biociências (DBCI), Universidade Federal de Sergipe (UFS), 49.500-000, Itabaiana-Sergipe, \\ Brasil \\ ${ }^{2}$ Universidade Federal do Vale do São Francisco (UNIVASF), 56300-000, Petrolina-Pernambuco, Brasil
}

*(julianofabricante@hotmail.com)

(Recebido em 14 de fevereiro de 2016; aceito em 21 de março de 2016)

\begin{abstract}
O estudo do banco de sementes (BS) pode ser utilizado como uma importante ferramenta para ponderar sobre o estado de conservação de espécies vegetais e de seus ambientes. Assim, o presente estudo objetivou avaliar o BS de sítios de Caatinga sob influência das obras do Projeto de Integração do Rio São Francisco com as Bacias Hidrográficas do Nordeste Setentrional (PISF). A área de estudo localizada em Sertânia, PE, foi dividida em três ambientes: Ambiente 1 - sítio de Caatinga alterado pelas obras do PISF; Ambiente 2 borda do fragmento de Caatinga; Ambiente 3 - interior do fragmento de Caatinga. A coleta dos solos foi feita em unidades amostrais de $20 \times 20 \mathrm{~cm}$, totalizando 20 amostras por ambiente. A avaliação do banco de sementes foi realizada pelo método de emergência de plântulas. Ao todo foram registradas 63 espécies, sendo 17 no Ambiente 1, 43 no Ambiente 2 e 44 no Ambiente 3. As desigualdades entre os ambientes observadas nesse estudo parecem ser reflexo direto das diferenças biofísicas existentes entre os sítios. Conclui-se que o BS dos sítios estudados apresenta diferenças significativas entre si na abundância e diversidade de espécies, especialmente em razão da grande quantidade de propágulos de espécies exóticas invasoras.
\end{abstract}

Palavras-chave: Invasão Biológica, Propágulos, Savana Estépica

The study of the seed bank (SB) can be used as an important tool to ponder the conservation status of plant species and their environments. Thus, this study aimed to evaluate the SB Caatinga sites under the influence of the works of the São Francisco River Integration Project with the Watershed of North Northeast (PWNN). The study area located in Sertânia, PE, was divided into three environment: Environment 1 Caatinga site changed by the works of PWNN; Environment 2 - edge of the Caatinga fragment; Environment 3 - Inside Caatinga fragment. The collection of soil was made in sample units of $20 \times 20 \mathrm{~cm}$, totaling 20 samples per environment. The evaluation of the seed bank was performed by emergency method of seedlings. In all, registered 63 species, 17 in Environment 1, 43 in Environment 2 and 44 in Environment 3. Inequalities between environments observed in this study appear to be a direct reflection of existing biophysical differences between the sites. It is concluded that the SB of the studied sites show significant differences among themselves in the abundance and diversity of species, especially given the large number of propagules of invasive alien species.

Keywords: Biological Invasion, Dry Forest, Propagules

\section{INTRODUÇÃO}

Historicamente grande parte da região Nordeste do Brasil é acometida por secas prolongadas, principalmente as regiões semiáridas cuja precipitação anual é de até $800 \mathrm{~mm}$. Visto a grande adversidade climática existente nessa região, foi criado pelo Governo Federal, sob responsabilidade do Ministério da Integração Nacional, o Projeto de Integração do Rio São Francisco com as Bacias Hidrográficas do Nordeste Setentrional (PISF) [1, 2]. Esse projeto tem como objetivo levar água do referido rio a outras regiões nordestinas por meio de dois canais: o Eixo Norte, que se estende por mais de $400 \mathrm{~km}$, e o Eixo Leste, que possui $220 \mathrm{~km}$ de extensão [1]. 
Para a realização dessa obra, uma área de mais de 12.000 ha $\left(120 \mathrm{~km}^{2}\right)$ está sendo completamente alterada [3], em uma região que possui 1.031 espécies vegetais [4]. Além da supressão da vegetação da área citada, as modificações nos solos e na hidrografia estão causando impactos importantes sobre as populações e comunidades vegetais da região, contudo, são poucas as informações sobre esses efeitos (Fabricante, J.R. - observação pessoal).

O estudo do banco de sementes (BS) pode ser uma importante ferramenta para ponderar sobre o estado de conservação de espécies vegetais e de seus ambientes. Seu conhecimento também pode contribuir para o entendimento do processo de sucessão ecológica local, e também auxiliar na busca de alternativas de baixo custo em projetos de recuperação de áreas degradadas [5]. Segundo Roberts (1981) [6], o BS é definido como a reserva de propágulos viáveis presentes no solo. Sendo assim, é um dos principais mecanismos para a recolonização da vegetação em um ambiente perturbado e é essencial para o equilíbrio dinâmico da área [7].

Em razão da importância do BS para conservação de populações autóctones e seus ecossistemas e a ausência de informações acerca desse tema em áreas de Caatinga sob influência das obras do PISF, o presente estudo objetivou avaliar esse atributo em diferentes sítios em uma dessas áreas.

\section{MATERIAL E MÉTODOS}

A área de estudo (89'17,7’'S; 37³0’35,3”W - $503 \mathrm{~m}$ altitude média) localiza-se no Eixo Leste do PISF, no município de Sertânia, PE. O clima regional é o BSh (semiárido quente) segundo a classificação de Köppen-Geiger, com precipitação anual de $635 \mathrm{~mm}$ e temperatura média de $25^{\circ} \mathrm{C}$ [8]. Ainda de acordo com CPRM (2005) [8], existem seis tipos de classes de solos no município, predominando os Luvissolos. A vegetação é composta pela Caatinga (Savana Estépica) em diferentes condições de conservação.

Para a realização desse estudo a área foi dividida em três ambientes, a saber:

Ambiente 1 - sítio de Caatinga alterado pelas obras do PISF. Nesse local a vegetação foi totalmente suprimida a cerca de três anos para a construção do canal do referido projeto e atualmente é dominado por espécies exóticas invasoras, especialmente por Nicotiana glauca Graham. Os solos encontram-se visualmente compactados e tiveram soterrados ou removidos seus horizontes iniciais $(\mathrm{O}$ e $\mathrm{A})$;

Ambiente 2 - borda do fragmento de Caatinga. Considerou-se como borda a região de transição entre o Ambiente 1 e o Ambiente 3, representada por uma faixa de 0-5 m de vegetação;

Ambiente 3 - interior do fragmento de Caatinga. Considerou-se como interior do fragmento pontos distantes pelo menos 20-30 $\mathrm{m}$ da faixa de borda do fragmento.

A coleta dos solos foi realizada em unidades amostrais com dimensões de $20 \mathrm{~cm}$ x $20 \mathrm{~cm}$ [9], totalizando 20 amostras por ambiente. As amostras foram coletadas a uma profundidade de 0-10 $\mathrm{cm}$, desprezando-se a camada de serapilheira. Os solos foram dispostos em bandejas de alumínio que foram mantidas em casa de vegetação totalmente isolada por sombrite de malha fina (30\%). Diariamente as amostras foram irrigadas e os solos foram revolvidos duas vezes durante o período do experimento.

A avaliação do BS foi realizada pelo método de emergência de plântulas [10], fazendo-se o monitoramento por um período de seis meses. A identificação das espécies foi realizada através de comparação com material testemunha existente no Herbário Vale do São Francisco (HVASF), Universidade Federal do Vale do São Francisco (UNIVASF), Petrolina-PE e por meio de consultas a especialistas. Toda espécie com material reprodutivo foi coletada, herborizada e incorporada ao acervo do referido herbário. A classificação taxonômica foi elaborada de acordo com o Sistema APG III (2009) [11]. 
Para cada espécie foram calculados os valores absolutos e relativos de densidade, frequência e valor de importância, sendo esse último obtido pela somatória das densidades e frequências relativas. A diversidade do BS foi avaliada por meio do índice de Shannon-Weaver $\left(H^{\prime}\right)$ [12], e a equabilidade através do índice de Pielou (E) [13]. Para avaliar a similaridade entre os ambientes foi utilizado o coeficiente de Jaccard $(S j)$ [14] e a dissimilaridade foi verificada pela distância Euclidiana [15].

Diferenças entre as diversidades foram verificadas pelo teste $\mathrm{t}(p \leq 0,05)$ [16]. A avaliação do grau de ajuste dos agrupamentos formados nas análises de similaridade e dissimilaridade foi verificada pelo coeficiente de correlação cofenética (ccc) conforme proposto por Sokal e Rohlf (1962) [17]. Já a validade dos agrupamentos foi determinada por meio do teste de permutação ANOSIM (oneway) [18]. As análises estatísticas foram executadas utilizando-se os softwares Past 2.17@ [19], MVSP 3.1@ [20] e BioEstat 5.3@ [21].

\section{RESULTADOS}

Ao todo foram registradas 63 espécies, sendo 17 no Ambiente 1, 43 no Ambiente 2 e 44 no Ambiente 3. Dentre as espécies amostradas, apenas Acalypha poiretii Spreng., Ageratum conyzoides L., Alternanthera tenella Colla, Eragrostis tenella (L.) P.Beauv. ex Roem. \& Schult., Euphorbia hirta L., Mollugo verticillata L., Nicotiana glauca Graham, Portulaca umbraticola Kunth e Sida sp. foram observadas nos três ambientes, onde juntas, representaram 14,3\% das espécies amostradas (Tabela 1).

A espécie com maior valor de importância (VI) no Ambiente 1 foi a exótica invasora $N$. glauca $(60,73 \%)$ devido a sua alta densidade (1.865 indivíduos $\left./ \mathrm{m}^{2}-90,7 \%\right)$ e presença em $100 \%$ das unidades amostrais. A segunda espécie com maior VI nesse Ambiente foi outra exótica invasora, E. tenella $(14,3 \%)$, que apresentou uma densidade de 113,7 indivíduos $/ \mathrm{m}^{2}(5,53 \%)$ e estava presente em $75 \%$ das amostras. Essas mesmas espécies foram as que apresentaram os maiores VI no Ambiente 2, contudo, E. tenella foi a mais importante $(26,81 \%)$, especialmente em razão da sua maior densidade $\left(516,2\right.$ indivíduos $\left./ \mathrm{m}^{2}-44,03 \%\right)$ em comparação com a da $N$. glauca (261,2 indivíduos $/ \mathrm{m}^{2}$ - 22,28\%). Já no Ambiente 3 uma espécie nativa, A. poiretii foi a que apresentou o maior VI $(13,02 \%)$, seguida das duas exóticas invasoras: E. tenella $(10,76 \%)$ e $N$. glauca $(8,99 \%)$.

Apesar das semelhanças apresentadas entre os Ambientes quanto às espécies com maior VI, observou-se que no Ambiente 3 houve uma distribuição mais homogenia desse parâmetro entre as espécies e uma diminuição na importância das exóticas. Enquanto que no Ambiente 1 e 2, $N$. glauca e E. tenella foram responsáveis por $75,04 \%$ e $43,96 \%$ do VI respectivamente, no Ambiente 3 esse valor foi de apenas 19,75\%.

A maior diversidade foi observada para o Ambiente $3\left(H^{\prime}=3,02\right)$, seguido do Ambiente $2\left(H^{\prime}\right.$ $=1,99)$ e do Ambiente $1\left(H^{\prime}=0,43\right)$. Segundo o teste t existem diferenças significativas entre as diversidades dos Ambientes (Ambiente $1 \times$ Ambiente $2-\mathrm{t}=26,6 ; \mathrm{p}<0,001$; Ambiente $1 \mathrm{x}$ Ambiente $3-\mathrm{t}=31,6 ; \mathrm{p}<0,001$; Ambiente $2 \times$ Ambiente $3-\mathrm{t}=10,8 ; \mathrm{p}<0,001)$. O índice de Pielou (E) foi de 0,15 para o Ambiente 1, de 0,53 para o Ambiente 2 e de 0,8 para o Ambiente 3.

Cinco espécies eram exclusivas do Ambiente 1, enquanto 13 foram observadas apenas no Ambiente 2, assim como no Ambiente 3. Os três ambientes compartilharam apenas nove espécies, já o número de espécies compartilhadas apenas entre os Ambientes 1 e 2 foi uma, entre os Ambientes 1 e 3 foram dois táxons e, entre os Ambientes 2 e 3 foram 20 espécies (Tabela 1). Desta forma, o maior índice de Jaccard $(S j)$ foi obtido entre os Ambientes 2 e $3(S j=0,5)$, contudo, $\mathrm{o}$ teste de permutação $\operatorname{ANOSIM}(\mathrm{R}=0,185 ; \mathrm{p}<0,001)$ revelou não existir similaridade florística entre os mesmos, assim como entre eles e o Ambiente 1 (Tabela 2). A menor dissimilaridade também foi observada entre os mesmos Ambientes (2 e 3); segundo a análise de $\operatorname{ANOSIM}(\mathrm{R}=0,322 ; \mathrm{p}<0,001)$ há dissimilaridade entre todos os agrupamentos (Tabela 3$)$. 
Tabela 1: Estrutura do banco de sementes nos Ambientes estudados, Sertânia-PE. Sendo: Ambiente 1 - sítio de Caatinga alterado pelas obras do PISF; Ambiente 2 borda do fragmento de Caatinga; Ambiente 3 - interior do fragmento de Caatinga; DA - densidade absoluta $\left(\mathrm{m}^{2}\right)$; DR - densidade relativa (\%); FA - frequência absoluta (\%); FR - frequência relativa (\%); VI - valor de importância; VI\% - porcentagem do valor de importância; Voucher - número de registro das coletas no Herbário Vale do São Francisco (HVASF/ UNIVASF).

\begin{tabular}{|c|c|c|c|c|c|c|c|c|c|c|c|c|c|c|c|c|c|c|c|}
\hline \multirow[t]{2}{*}{ Espécies } & \multicolumn{6}{|c|}{ Ambiente 1} & \multicolumn{6}{|c|}{ Ambiente 2} & \multicolumn{6}{|c|}{ Ambiente 3} & \multirow[t]{2}{*}{ Voucher } \\
\hline & DA & DR & FA & FR & VI & $\% \mathrm{VI}$ & $\mathrm{DA}$ & DR & FA & FR & VI & $\% \mathrm{VI}$ & DA & DR & FA & FR & VI & $\% \mathrm{VI}$ & \\
\hline Nicotiana glauca Graham & 1865 & 90,7 & 100 & 30,77 & 121,5 & 60,73 & 261,2 & 22,28 & 75 & 12 & 34,28 & 17,14 & 31,25 & 9,65 & 50 & 8,33 & 17,99 & 8,99 & 14326 \\
\hline $\begin{array}{l}\text { Eragrostis tenella (L.) } \\
\text { P.Beauv. ex Roem. \&Schult. }\end{array}$ & 113,7 & 5,53 & 75 & 23,08 & 28,61 & 14,3 & 516,2 & 44,03 & 60 & 9,6 & 53,63 & 26,81 & 40 & 12,36 & 55 & 9,17 & 21,52 & 10,76 & 22446 \\
\hline Acalypha poiretii Spreng. & 43,75 & 2,13 & 55 & 16,92 & 19,05 & 9,53 & 16,25 & 1,39 & 30 & 4,8 & 6,19 & 3,09 & 60 & 18,53 & 45 & 7,5 & 26,03 & 13,02 & 22448 \\
\hline Euphorbia hirta L. & 12,5 & 0,61 & 30 & 9,23 & 9,84 & 4,92 & 82,5 & 7,04 & 65 & 10,4 & 17,44 & 8,72 & 1,25 & 0,39 & 5 & 0,83 & 1,22 & 0,61 & 22489 \\
\hline $\begin{array}{l}\text { Desmodium tortuosum (Sw.) } \\
\text { DC. }\end{array}$ & & & & & & & 12,5 & 1,07 & 15 & 2,4 & 3,47 & 1,73 & 31,25 & 9,65 & 30 & 5 & 14,65 & 7,33 & 22486 \\
\hline Mollugo verticillata $\mathrm{L}$. & 1,25 & 0,06 & 5 & 1,54 & 1,6 & 0,8 & 90 & 7,68 & 20 & 3,2 & 10,88 & 5,44 & 2,5 & 0,77 & 10 & 1,67 & 2,44 & 1,22 & 22445 \\
\hline Ageratum conyzoides $\mathrm{L}$. & 1,25 & 0,06 & 5 & 1,54 & 1,6 & 0,8 & 38,75 & 3,3 & 45 & 7,2 & 10,5 & 5,25 & 2,5 & 0,77 & 10 & 1,67 & 2,44 & 1,22 & 22497 \\
\hline Sida sp. & 1,25 & 0,06 & 5 & 1,54 & 1,6 & 0,8 & 16,25 & 1,39 & 25 & 4 & 5,39 & 2,69 & 7,5 & 2,32 & 25 & 4,17 & 6,48 & 3,24 & 22461 \\
\hline Oxalis glaucescens Norlind & & & & & & & 6,25 & 0,53 & 20 & 3,2 & 3,73 & 1,87 & 15 & 4,63 & 25 & 4,17 & 8,8 & 4,4 & 22449 \\
\hline Ocimum campechianum Mill. & & & & & & & 6,25 & 0,53 & 15 & 2,4 & 2,93 & 1,47 & 11,25 & 3,47 & 25 & 4,17 & 7,64 & 3,82 & 22450 \\
\hline Alternanthera tenella Colla & 2,5 & 0,12 & 5 & 1,54 & 1,66 & 0,83 & 2,5 & 0,21 & 5 & 0,8 & 1,01 & 0,51 & 11,25 & 3,47 & 25 & 4,17 & 7,64 & 3,82 & 22456 \\
\hline Tarenaya spinosa (Jacq.) Raf. & & & & & & & 3,75 & 0,32 & 15 & 2,4 & 2,72 & 1,36 & 13,75 & 4,25 & 20 & 3,33 & 7,58 & 3,79 & - \\
\hline $\begin{array}{l}\text { Selaginella convoluta (Arn.) } \\
\text { Spring }\end{array}$ & & & & & & & 12,5 & 1,07 & 5 & 0,8 & 1,87 & 0,93 & 10 & 3,09 & 20 & 3,33 & 6,42 & 3,21 & 22511 \\
\hline Croton sp. & & & & & & & 5 & 0,43 & 10 & 1,6 & 2,03 & 1,01 & 8,75 & 2,7 & 20 & 3,33 & 6,04 & 3,02 & - \\
\hline $\begin{array}{l}\text { Enteropogon mollis (Ness) } \\
\text { Clayton }\end{array}$ & & & & & & & 18,75 & 1,6 & 15 & 2,4 & 4 & 2 & 6,25 & 1,93 & 10 & 1,67 & 3,6 & 1,8 & 22501 \\
\hline Cyperus uncilatus Poir. & & & & & & & 5 & 0,43 & 5 & 0,8 & 1,23 & 0,61 & 7,5 & 2,32 & 20 & 3,33 & 5,65 & 2,82 & 22512 \\
\hline Portulaca umbraticola Kunth & 1,25 & 0,06 & 5 & 1,54 & 1,6 & 0,8 & 5 & 0,43 & 15 & 2,4 & 2,83 & 1,41 & 2,5 & 0,77 & 5 & 0,83 & 1,61 & 0,8 & 22452 \\
\hline $\begin{array}{l}\text { Borreria spinosa (L.) Cham. \& } \\
\text { Schltdl }\end{array}$ & & & & & & & 5 & 0,43 & 10 & 1,6 & 2,03 & 1,01 & 3,75 & 1,16 & 10 & 1,67 & 2,82 & 1,41 & 22463 \\
\hline Bidens pilosa $\mathrm{L}$. & & & & & & & & & & & & & 7,5 & 2,32 & 15 & 2,5 & 4,82 & 2,41 & 22493 \\
\hline $\begin{array}{l}\text { Myracroduon } \\
\text { Allemão }\end{array}$ & & & & & & & 8,75 & 0,75 & 25 & 4 & 4,75 & 2,37 & & & & & & & - \\
\hline Ruellia sp. & & & & & & & 1,25 & 0,11 & 5 & 0,8 & 0,91 & 0,45 & 3,75 & 1,16 & 15 & 2,5 & 3,66 & 1,83 & 22507 \\
\hline $\begin{array}{l}\text { Dactyloctenium aegyptium (L.) } \\
\text { Willd. }\end{array}$ & 1,25 & 0,06 & 5 & 1,54 & 1,6 & 0,8 & & & & & & & 2,5 & 0,77 & 10 & 1,67 & 2,44 & 1,22 & 22460 \\
\hline
\end{tabular}




\begin{tabular}{|c|c|c|c|c|c|c|c|c|c|c|c|c|c|c|c|c|c|c|c|}
\hline Phylanthus niruri L. & & & & & & & 1,25 & 0,11 & 5 & 0,8 & 0,91 & 0,45 & 3,75 & 1,16 & 10 & 1,67 & 2,82 & 1,41 & 22447 \\
\hline $\begin{array}{l}\text { Herissantia } \quad \text { crispa } \\
\text { Brizicky }\end{array}$ & & & & & & & 8,75 & 0,75 & 10 & 1,6 & 2,35 & 1,17 & 1,25 & 0,39 & 5 & 0,83 & 1,22 & 0,61 & 22466 \\
\hline Diodella teres (Walter) Small & 2,5 & 0,12 & 5 & 1,54 & 1,66 & 0,83 & 2,5 & 0,21 & 10 & 1,6 & 1,81 & 0,91 & & & & & & & 22458 \\
\hline Evolvulus filipes Mart. & & & & & & & 1,25 & 0,11 & 5 & 0,8 & 0,91 & 0,45 & 2,5 & 0,77 & 10 & 1,67 & 2,44 & 1,22 & 22488 \\
\hline Spigelia anthelmia $\mathrm{L}$. & & & & & & & 1,25 & 0,11 & 5 & 0,8 & 0,91 & 0,45 & 2,5 & 0,77 & 10 & 1,67 & 2,44 & 1,22 & 22457 \\
\hline $\begin{array}{l}\text { Justicia angustissima A.L.A. } \\
\text { Côrtes \& Rapini }\end{array}$ & & & & & & & & & & & & & 5 & 1,54 & 10 & 1,67 & 3,21 & 1,61 & 22490 \\
\hline $\begin{array}{l}\text { Centrosema brasilianum (L.) } \\
\text { Benth. }\end{array}$ & & & & & & & 2,5 & 0,21 & 10 & 1,6 & 1,81 & 0,91 & 1,25 & 0,39 & 5 & 0,83 & 1,22 & 0,61 & 22478 \\
\hline Merremia aegyptia (L.) Urb. & & & & & & & 2,5 & 0,21 & 10 & 1,6 & 1,81 & 0,91 & 1,25 & 0,39 & 5 & 0,83 & 1,22 & 0,61 & 22470 \\
\hline Croton blachetianus Baill. & & & & & & & & & & & & & 3,75 & 1,16 & 10 & 1,67 & 2,82 & 1,41 & - \\
\hline $\begin{array}{l}\text { Oxalis divaricata Mart. ex } \\
\text { Zucc. }\end{array}$ & & & & & & & & & & & & & 3,75 & 1,16 & 10 & 1,67 & 2,82 & 1,41 & 22451 \\
\hline Paspalum arenarium Schrad. & 1,25 & 0,06 & 5 & 1,54 & 1,6 & 0,8 & & & & & & & 1,25 & 0,39 & 5 & 0,83 & 1,22 & 0,61 & 22475 \\
\hline $\begin{array}{l}\text { Conyza bonariensis } \quad \text { (L.) } \\
\text { Cronquist }\end{array}$ & & & & & & & & & & & & & 2,5 & 0,77 & 10 & 1,67 & 2,44 & 1,22 & 22505 \\
\hline Cyperus sp. & & & & & & & 3,75 & 0,32 & 5 & 0,8 & 1,12 & 0,56 & 1,25 & 0,39 & 5 & 0,83 & 1,22 & 0,61 & 22455 \\
\hline $\begin{array}{l}\text { Malvastrum tomentosum (L.) } \\
\text { S.R. Hill }\end{array}$ & & & & & & & 7,5 & 0,64 & 10 & 1,6 & 2,24 & 1,12 & & & & & & & 22487 \\
\hline $\begin{array}{l}\text { Asemeia ovata (Poir.) } \\
\text { J.F.B.Pastore \& J.R.Abbott }\end{array}$ & & & & & & & 1,25 & 0,11 & 5 & 0,8 & 0,91 & 0,45 & 1,25 & 0,39 & 5 & 0,83 & 1,22 & 0,61 & 22459 \\
\hline $\begin{array}{l}\text { Bernardia sidoides (Klotzsch) } \\
\text { Müll.Arg. }\end{array}$ & & & & & & & 1,25 & 0,11 & 5 & 0,8 & 0,91 & 0,45 & 1,25 & 0,39 & 5 & 0,83 & 1,22 & 0,61 & 22462 \\
\hline Centratherum punctatum Cass. & & & & & & & 1,25 & 0,11 & 5 & 0,8 & 0,91 & 0,45 & 1,25 & 0,39 & 5 & 0,83 & 1,22 & 0,61 & 22481 \\
\hline $\begin{array}{l}\text { Commiphora leptophloeos } \\
\text { (Mart.) J.B.Gillet }\end{array}$ & & & & & & & 3,75 & 0,32 & 10 & 1,6 & 1,92 & 0,96 & & & & & & & - \\
\hline Chenopodium ambrosioides L. & 2,5 & 0,12 & 5 & 1,54 & 1,66 & 0,83 & & & & & & & & & & & & & 22513 \\
\hline Eclipta próstata (L.) L. & 2,5 & 0,12 & 5 & 1,54 & 1,66 & 0,83 & & & & & & & & & & & & & 22471 \\
\hline $\begin{array}{l}\text { Digitaria cf. ciliaris (Retz.) } \\
\text { Koeler }\end{array}$ & 1,25 & 0,06 & 5 & 1,54 & 1,6 & 0,8 & & & & & & & & & & & & & 22485 \\
\hline $\begin{array}{l}\text { Eragrostis vernix Boechat \& } \\
\text { Longhi-Wagner }\end{array}$ & 1,25 & 0,06 & 5 & 1,54 & 1,6 & 0,8 & & & & & & & & & & & & & - \\
\hline Euphorbia prostrata Aiton & 1,25 & 0,06 & 5 & 1,54 & 1,6 & 0,8 & & & & & & & & & & & & & 22454 \\
\hline $\begin{array}{l}\text { Tacinga inamoena (K.Schum.) } \\
\text { N.P.Taylor \& Stuppy }\end{array}$ & & & & & & & 8,75 & 0,75 & 5 & 0,8 & 1,55 & 0,77 & & & & & & & - \\
\hline
\end{tabular}




\begin{tabular}{|c|c|c|c|c|c|c|c|c|c|c|c|c|c|c|c|c|c|c|}
\hline $\begin{array}{l}\text { Callisia filiformis (M.Martens } \\
\text { \& Galeotti) D.R.Hunt }\end{array}$ & & & & & & & & & & & & 1,25 & 0,39 & 5 & 0,83 & 1,22 & 0,61 & 22465 \\
\hline Corchorus hirtus L. & & & & & & & & & & & & 1,25 & 0,39 & 5 & 0,83 & 1,22 & 0,61 & 22468 \\
\hline Cynodon dactylon (L.) Pers. & & & & & & & & & & & & 1,25 & 0,39 & 5 & 0,83 & 1,22 & 0,61 & 22464 \\
\hline Delilia biflora (L.) Kuntze & & & & & & & & & & & & 1,25 & 0,39 & 5 & 0,83 & 1,22 & 0,61 & 22491 \\
\hline Fimbristylis miliacea (L.) Vahl & & & & & & & & & & & & 1,25 & 0,39 & 5 & 0,83 & 1,22 & 0,61 & 22480 \\
\hline Gnaphalium polycaulon Pers. & & & & & & & & & & & & 1,25 & 0,39 & 5 & 0,83 & 1,22 & 0,61 & 22469 \\
\hline $\begin{array}{l}\text { Ludwigia cf. affinis (DC.) H. } \\
\text { Hara }\end{array}$ & & & & & & & & & & & & 1,25 & 0,39 & 5 & 0,83 & 1,22 & 0,61 & 22494 \\
\hline Poaceae & & & & & & & & & & & & 1,25 & 0,39 & 5 & 0,83 & 1,22 & 0,61 & 22474 \\
\hline Cnidosculus urens (L.) Arthur & & & & & & 1,25 & 0,11 & 5 & 0,8 & 0,91 & 0,45 & & & & & & & 22509 \\
\hline $\begin{array}{l}\text { Dioclea grandiflora Mart. ex } \\
\text { Benth. }\end{array}$ & & & & & & 1,25 & 0,11 & 5 & 0,8 & 0,91 & 0,45 & & & & & & & - \\
\hline $\begin{array}{l}\text { Macroptilium lathyroides (L.) } \\
\text { Urb. }\end{array}$ & & & & & & 1,25 & 0,11 & 5 & 0,8 & 0,91 & 0,45 & & & & & & & 22473 \\
\hline $\begin{array}{l}\text { Paspalum scutatum Nees ex } \\
\text { Trin. }\end{array}$ & & & & & & 1,25 & 0,11 & 5 & 0,8 & 0,91 & 0,45 & & & & & & & 22467 \\
\hline $\begin{array}{l}\text { Poincianella microphylla } \\
\text { (Mart. ex G.Don) L.P.Queiroz }\end{array}$ & & & & & & 1,25 & 0,11 & 5 & 0,8 & 0,91 & 0,45 & & & & & & & - \\
\hline Schinopsis brasiliensis Engl. & & & & & & 1,25 & 0,11 & 5 & 0,8 & 0,91 & 0,45 & & & & & & & - \\
\hline Tragia volubilis $\mathrm{L}$. & & & & & & 1,25 & 0,11 & 5 & 0,8 & 0,91 & 0,45 & & & & & & & 22477 \\
\hline Waltheria rotundifolia Schrank & & & & & & 1,25 & 0,11 & 5 & 0,8 & 0,91 & 0,45 & & & & & & & 22476 \\
\hline $\begin{array}{l}\text { Wissadula caribea (A.DC.) } \\
\text { Bovini }\end{array}$ & & & & & & 1,25 & 0,11 & 5 & 0,8 & 0,91 & 0,45 & & & & & & & 22498 \\
\hline Total & $2056 \quad 100$ & 325 & 100 & 200 & 100 & 1172 & 100 & 625 & 100 & 200 & 100 & 323,8 & 100 & 600 & 100 & 200 & 100 & \\
\hline
\end{tabular}


Tabela 2: Similaridade de Jaccard (Sj) entre os Ambientes estudados, Sertânia-PE. Sendo: Ambiente 1 - sítio de Caatinga alterado pelas obras do PISF; Ambiente 2 - borda do fragmento de Caatinga; Ambiente 3 - interior do fragmento de

Caatinga. As células em branco representam os valores de Sj e as células em cinza representam a probabilidade do teste de ANOSIM. Coeficiente de correlação cofenética $=0,998$.

\begin{tabular}{llll}
\hline & Ambiente 1 & Ambiente 2 & Ambiente 3 \\
\hline Ambiente 1 & & 0,0001 & 0,0001 \\
Ambiente 2 & 0,2 & & 0,0045 \\
Ambiente 3 & 0,22 & 0,5 & \\
\hline
\end{tabular}

Tabela 3: Distância Euclidiana entre os Ambientes estudados, Sertânia-PE. Sendo: Ambiente 1 - sítio de Caatinga alterado pelas obras do PISF; Ambiente 2 - borda do fragmento de Caatinga; Ambiente 3 - interior do fragmento de Caatinga. As células em branco representam os valores da Distância Euclidiana e as células em cinza representam a probabilidade do teste de ANOSIM. Coeficiente de correlação cofenética =0,991.

\begin{tabular}{llll}
\hline & Ambiente 1 & Ambiente 2 & Ambiente 3 \\
\hline Ambiente 1 & & 0,0001 & 0,0001 \\
Ambiente 2 & 1326,8 & & 0,001 \\
Ambiente 3 & 1468,7 & 437,1 & \\
\hline
\end{tabular}

\section{DISCUSSÃO}

O número de espécies amostradas nesse trabalho foi inferior aos encontrados nos estudos de Gonçalves et al. (2011) [22] e Parente et al. (2011) [23], e superior aos de Costa e Araújo (2003) [24], Mamede e Araújo (2008) [25] e Santos et al. (2010) [26]. Outro aspecto observado foi a presença de várias espécies exclusivas em cada estudo, demonstrando assim a grande variação espacial do BS em áreas de Caatinga.

Entre os ambientes estudados a variação na riqueza e na composição especifica também foi verificada. As desigualdades parecem ser reflexo direto das diferenças biofísicas existentes entre os sítios. A supressão da vegetação e a remoção ou soterramento das camadas superficiais dos solos do Ambiente 1, além de eliminar parte importante do BS existente antes das obras, também formaram nichos bem distintos dos originais (ausência de cobertura vegetal, insolação intensa, maior temperatura, menor umidade, maior exposição as correntes de ar e solos extremamente compactados e pouco permeáveis). Esse conjunto de condições parece também refletir sobre a abundância das espécies do BS, favorecendo especialmente as exóticas, as quais formaram grandes reservatórios de propágulos. Alguns autores argumentam que baixa riqueza de espécies e ou a existência de distúrbios são importantes fatores facilitadores das invasões biológicas [27, 28, 29].

A abundância das duas exóticas no Ambiente 1 e consecutiva diminuição nos outros ambientes, refletiram sobre os valores de equabilidade dos sítios que, associado as diferenças na riqueza de espécies, promoveram as desigualdades observadas entre as diversidades dos ambientes, sendo menor no Ambiente 1, intermediária no Ambiente 2 e maior no Ambiente 3. Os valores de diversidade encontrados nos Ambientes 1 e 2 foram comparativamente inferiores aos apresentados por [23] e [22], os quais por sua vez foram semelhantes ao do Ambiente 3.

Outra diferença observada nesse estudo foi a diminuição na densidade do BS no sentido do ambiente mais degradado (Ambiente 1) para o ambiente mais conservado (Ambiente 3). Esses resultados corroboram com a ideia de que a abundância de sementes tende a ser maior em ambientes em fases iniciais de sucessão [30, 31, 32].

Apesar do desenho experimental não permitir concluir, os resultados desse estudo sugerem haver um efeito de borda sobre o BS local. Essa comprovação refutaria os resultados de outros estudos que concluíram que esse efeito (se existir) não altera o componente biológico da Caatinga [26, 33]. 
A despeito da diminuição da densidade de $N$. glauca no Ambiente 3, sua presença ali é bastante preocupante, uma vez que trata-se do interior de um fragmento de Caatinga. O número de espécimes observado nesse Ambiente corresponde a uma densidade de 312.500 indivíduos ha ${ }^{-1}$. Além de extremamente tóxica $[34,35], N$. glauca ainda causa impactos sobre os ambientes invadidos $[36,37,38$, 39] e desta forma pode comprometer a conservação das espécies autóctones. Além disso, muitas áreas semelhantes a estudada serão futuramente exploradas pela agricultura e pela pecuária, cujas conversões dos ambientes poderão favorecer a expansão das áreas invadidas por $N$. glauca e a importância da espécie na Região.

Conclui-se que o BS dos sítios estudados apresenta diferenças significativas entre si na abundância e diversidade de espécies, especialmente em razão da grande quantidade de propágulos de espécies exóticas invasoras. Os nichos criados pelas obras do PISF parecem servir como agentes facilitadores para a dispersão dessas espécies, o que torna necessário e urgente o seu manejo.

\section{AGRADECIMENTOS}

Os autores agradecem ao Ministério da Integração Nacional (MI), aos Analistas Ambientais do Núcleo de Ecologia e Monitoramento Ambiental (NEMA/UNIVASF) pela auxilio na identificação do material botânico e ao Centro de Referência para Recuperação de Áreas

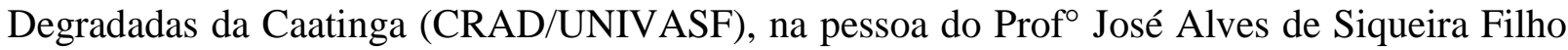
pelo apoio logístico.

\section{REFERÊNCIAS BIBLIOGRÁFICAS}

1. MI. Projeto de Integração do Rio São Francisco com Bacias Hidrográficas do Nordeste Setentrional. 2004. 129 p. (Relatório de Impacto Ambiental).

2. Castro CN. Transposição do Rio São Francisco: análise de oportunidade do projeto. Brasília: IPEA; 2011. 60 p.

3. Fabricante JR, Siqueira-Filho JA. Mudanças nas paisagens das caatingas e as invasões biológicas. Boletín de la Red Latinoamericana para el Estudio de Plantas Invasoras. 2012 Jul;2:23-27.

4. Siqueira-Filho JA et al. (79 coautores). Flora das Caatingas do Rio São Francisco. Rio de Janeiro (Brasil): Andrea Jakobsson Estúdio Editorial; 2012. Capítulo 13, Flora das Caatingas do Rio São Francisco: história natural e conservação; p. 446-542.

5. Cabral MA, Queiroz SEE. Uso do banco de sementes do solo como indicativo para recuperação de áreas degradadas. Revista de Biologia e Ciências da Terra. 2012;12(1):43-48.

6. Roberts HA. Seed banks in the soil. Cambridge (England): Academic Press; 1981. Advances in Applied Biology; p. 55.

7. Schmitz MC. Banco de sementes no solo em áreas do reservatório da UHE Paraibuna. Piracicaba (Brasil): SÉRIE IPEF; 1992. Recomposição da vegetação com espécies arbóreas nativas em reservatórios de usinas hidrelétricas da CESP; p. 7-8.

8. CPRM. Projeto cadastro de fontes de abastecimento por água subterrânea - Diagnóstico do município de Sertânia, estado de Pernambuco. Recife: CPRM/PRODEEM; 2005. 12 p.

9. Thompson K. Small-scale heterogeneity in the seed bank of an acidic grassland. Journal of Ecology. 1986 Sep;74:733-738, doi: 10.2307/2260394.

10. Santos DM, Silva KA, Santos JMFF, Lopes CGR, Pimentel RMM, Araújo EL. Variação espaço-temporal do banco de sementes em uma área de floresta tropical seca (Caatinga) - Pernambuco. Revista de Geografia. 2010 Apr/Jan;27(1):234-253.

11. APG III. An update of the Angiosperm Phylogeny Group classification for the orders and families of flowering plants: APG III. Botanical Journal of the Linnean Society. 2009 Oct;161:105-121, doi: 10.1111/j.10958339.2009.00996.x

12. Shannon CE, Weaver W. The Mathematical Theory of Communication. Urbana: University Illinois Press. 1949. $117 \mathrm{p}$.

13. Pielou UC. Mathematical diversity. New York: John Wiley e Sons, 1977. 385 p.

14. Mueller-Dombois D, Ellenberg H. Aims and methods of vegetation ecology. New York: John Wiley \& Sons, 1974. $547 \mathrm{p}$. 
15. Brower JE, Zar JH. Field and laboratory methods for general ecology. Dubuque: WMC Brown, 1984. 288 p.

16. Lehmann EL. Testing statistical hypotheses. 2 ed. New York: Springer-Verlag, 1997. 352 p.

17. Sokal RR, Rohlf FJ. The comparison of dendrograms by objective methods. Taxon. 1962 Feb;11(2):30-40, doi: $10.2307 / 1217208$.

18. Clarke KR. Non-parametric multivariate analyses of changes in community structure. Australian Journal of Ecology. 1993 Jul;18:117-143,doi:10.1111/j.1442-9993.1993.tb00438.x.

19. Hammer $\varnothing$, Harper DAT, Ryan PD. Past: Paleontological Statistics Software package for education and data analysis. Palaeontologia Electronica. 2001 Jun;4(1):1-9, doi: 10.1016/j.bcp.2008.05.025.

20. Kovach WL. MVSP: A MultiVariate Statistical Package for Windows, ver. 3.1. Pentraeth: Kovach Computing Services, 2005. 137 p.

21. Ayres M, Ayres MJ, Ayres DL, Santos SA. Bioestat 5.3: aplicações estatísticas nas áreas das Ciências Biomédicas. Belém: Mamirauá/CNPq, 2007. 324 p.

22. Gonçalves GS, Andrade LA, Xavier KRF, Oliveira LSB, Moura MA. Estudo do banco de sementes do solo em uma área de Caatinga invadida por Parkinsonia aculeata L. Revista Brasileira de Biociências. Porto Alegre. 2011 Out/dez;9(4):428-436.

23. Parente RG, Barbosa LG, Souza OZ, Vilar FCR. Composição florística do banco de sementes do solo da Caatinga em perímetro irrigado de Petrolina - Pernambuco. Semiárido de Visu. 2011 May/Ago;1(1):18-31.

24. Costa RC, Araújo FS. Densidade, germinação e flora do banco de sementes do solo no final da estação seca, em uma área de caatinga, Quixadá, CE. Acta Botanica Brasilica. 2003 Apr/jun;17:259-264, doi: 10.1590/S010233061003000200008.

25. Mamede MA, Araújo FS. Effects of slash and burn practices on a soil seed bank of Caatinga vegetation in Northeastern Brazil. Journal of Arid Environments. 2008 Apr;72:458-470,doi: 10.1016/j.jaridenv.2007.07.014.

26. Santos AMM, Santos BA. Are the vegetation structure and composition of the shrubby Caatinga free from edge influence? Acta Botanica Braslica. 2008 Oct/Dec;22:1077-1084, doi: 10.1590/S0102-33062001000400018.

27. Williamson M. Biological invasions. London: Chapman \& Hall, 1996. 244 p.

28. Tillman D. Community invasibility, recruitment limitation, and grassland biodiversity. Ecology. 1997 Jan;78(1):81-92, doi: 10.2307/2265980.

29. Mack RN, Simberloff D, Lonsdale WM, Evans H, Clout M, Bazzaz F. Biotic invasions: causes, epidemiology, global consequences and control. Issues in Ecology. 2000 Jun;(5):1-20,doi: 10.1890/10510761(2000)010[0689:BICEGC]2.0.CO;2.

30. Garwood NC. Tropical soil seed banks, a review. Cambridge (England): Academic Press; 1989. Chapter 9, Ecology of soil seed banks; p. 149-209, doi: 10.1016/B978-0-12-440405-2.50014-2.

31. Araújo MM, Oliveira FA, Vieira ICG, Barros CLC, Lima CAT. Densidade e composição florística do banco de sementes do solo de florestas sucessionais na região do Baixo Rio Guamá, Amazônia Oriental. Scientia Forestalis. 2001 Jun;59:115-130.

32. Baider C, Tabarelli M, Mantovani W. O banco de sementes de um trecho de floresta atlântica montana (São Paulo, Brasil). Revista Brasileira de Biologia. 1999 May;59:319-328, doi: 10.1590/S003471081999000200014.

33. Oliveira DG, Prata APN, Souto LS, Ferreira RA. Does the edge effect influence plant community structure in a tropical dry forest? Revista Árvore. 2013 Apr;37:311-320, doi: 10.1590/S0100-67622013000200012.

34. Panter KE, Weinzweig J, Gardner DR, Stegelmeier BL, James LF. Comparison of cleft palate induction by Nicotiana glauca in goats and sheep. Teratology. 2000 Febr;61:203-210, 10.1002/(SICI)10969926(200003)61:3<203::AID-TERA8>3.0.CO;2-I.

35. Botha CJ, Steenkamp PA, Olivier A, Bekker LC. Nicotiana glauca poisoning in ostriches (Struthio camelus). Journal of the South African Veterinary Association. 2011 Jun;82(2):116-119, doi: 10.4102/jsava.v82i2.44.

36. Brandes D. Nicotiana glauca als invasive Pflanzeauf Fuerteventura. Dietmar Brandes (Hrsg.): Adventivpflamen; 2000. Beiträge zu Biologie, Vorkommen und Ausbreitungsdynamik von Archäophyten und Neophyten in Mitteleuropa; 39-57 p.

37. Sanz-Elorza M, Dana ED, Sobrino E. Atlas de lãs plantas alóctonas invasoras de España. Madrid: Dirección General para la Biodiversidad; 2004. 384 p.

38. Alshahrani ST. Effect of aqueous extract of the invasive species tobacco (Nicotiana glauca L.) on seedlings growth of Juniper (Juniperus procera L.). Emirates Journal of Food and Agriculture. 2008 Jul;20(2):10-17, doi: 10.9755/ejfa.v20i2.5186.

39. Fabricante JR, Araújo KCT, Castro RC, Siqueira-Filho JA. Nicotiana glauca Graham. Florianópolis: Bookes; 2013. Plantas exóticas e exóticas invasoras da Caatinga; p. 29-36. 